\title{
Dunkl Operators and Canonical Invariants of Reflection Groups ${ }^{\star}$
}

\author{
Arkady BERENSTEIN ${ }^{\dagger}$ and Yurii BURMAN $\ddagger \S$ \\ $\dagger$ Department of Mathematics, University of Oregon, Eugene, OR 97403, USA \\ E-mail: arkadiy@math.uoregon.edu \\ $\ddagger$ Independent University of Moscow, 11 B. Vlassievsky per., 121002 Moscow, Russia \\ E-mail: burman@mccme.ru \\ $\S$ Higher School of Economics, 20 Myasnitskaya Str., 101000 Moscow, Russia
}

Received December 14, 2008, in final form May 21, 2009; Published online June 03, 2009

doi:10.3842/SIGMA.2009.057

\begin{abstract}
Using Dunkl operators, we introduce a continuous family of canonical invariants of finite reflection groups. We verify that the elementary canonical invariants of the symmetric group are deformations of the elementary symmetric polynomials. We also compute the canonical invariants for all dihedral groups as certain hypergeometric functions.
\end{abstract}

Key words: Dunkl operators; reflection group

2000 Mathematics Subject Classification: 20F55; 15A72

\section{Introduction and main results}

Let $V_{\mathbb{R}}$ be a real vector space with a scalar product, and $W \subset O\left(V_{\mathbb{R}}\right)$ be a finite group generated by reflections. In this paper we construct a family of $W$-invariants (which we refer to as canonical invariants) in $S(V)$, where $V=\mathbb{C} \otimes V_{\mathbb{R}}$, by means of Dunkl operators (see [8]). These canonical invariants form a basis in $S(V)^{W}$ (depending on a continuous parameter $c$ ) and, as such, include both the $c$-elementary and $c$-quasiharmonic invariants introduced in our earlier paper [2]. Using this technique, we prove that for $W=S_{n}$ the $c$-elementary invariants are deformations of the elementary symmetric polynomials in the vicinity of $c=1 / n$.

Dunkl operators $\nabla_{y}, y \in V^{*}$, are differential-difference operators first introduced by Charles Dunkl in [8] and given (for any $W$ ) by:

$$
\nabla_{y}=\partial_{y}-\sum_{s \in S} c(s) \frac{\left\langle y, \alpha_{s}\right\rangle}{\alpha_{s}}(1-s),
$$

where $S$ is the set of all reflections in $W, c: S \rightarrow \mathbb{C}$ is a $W$-invariant function on $S$, and $\alpha_{s} \in V$ is the root of the reflection $s$. In particular, for $W=S_{n}$,

$$
\nabla_{y}=\partial_{y}-c \sum_{1 \leq i<j \leq n} \frac{y_{i}-y_{j}}{x_{i}-x_{j}}\left(1-s_{i j}\right)
$$

where $s_{i j} \in S_{n}$ is the transposition switching $x_{i}$ and $x_{j}$.

The remarkable result by Charles Dunkl that all $\nabla_{y}$ commute allows to define the operators $\nabla_{p}, p \in S\left(V^{*}\right)$, by $\nabla_{p+q}=\nabla_{p}+\nabla_{q}, \nabla_{p q}=\nabla_{p} \nabla_{q}$ for all $p, q \in S\left(V^{*}\right)$.

In what follows we will mostly think of $c$ as a formal parameter in the affine space $\mathbb{A}^{S / W}$, where $S / W$ is the set of $W$-orbits in $S$. Using the notation $S_{c}(V)=\mathbb{C}(c) \otimes S(V)$, consider each

\footnotetext{
${ }^{\star}$ This paper is a contribution to the Special Issue on Dunkl Operators and Related Topics. The full collection is available at http://www.emis.de/journals/SIGMA/Dunkl_operators.html
} 
Dunkl operator $\nabla_{p}$ as a $\mathbb{C}$-linear map $S(V) \rightarrow S_{c}(V)$ (or, extending scalars, as a $\mathbb{C}(c)$-linear endomorphism of $S_{c}(V)$ ).

Thus, the association $p \mapsto \nabla_{p}$ defines an action of $S_{c}\left(V^{*}\right)$ on $S_{c}(V)$ by differential-difference operators. In turn, this action and the isomorphism $V \cong V^{*}$ (hence $S_{c}\left(V^{*}\right) \cong S_{c}(V)$ ) given by the scalar product define the bilinear form $(\cdot, \cdot)_{c}: S_{c}(V) \times S_{c}(V) \rightarrow \mathbb{C}(c)$ by

$$
(f, g)_{c} \stackrel{\text { def }}{=}\left[\nabla_{f}(g)\right]_{0},
$$

for all $f, g \in S_{c}(V)$ where $x \mapsto[x]_{0}$ is the constant term projection $S_{c}(V) \rightarrow \mathbb{C}(c)$. Clearly, $(f, g)_{c}=0$ if $f, g$ are homogeneous and $\operatorname{deg} f \neq \operatorname{deg} g$.

The form (1.1) is symmetric and its specialization at generic $c: S \rightarrow \mathbb{C}$ and $c=0$ is nondegenerate. Understanding the values of $c$ when the specialization of the form is degenerate and the structure of the radical is crucial for the study of representations of the rational Cherednik algebra $H_{c}(W)$ (see e.g. $[9,11,4]$ ).

A classical Chevalley theorem [6] says that the algebra $S(V)^{W}$ of $W$-invariants in $S(V)$ is isomorphic to the algebra of polynomials $\mathbb{C}\left[u_{1}, \ldots, u_{\ell}\right]$ of certain homogeneous elements $u_{1}, \ldots, u_{\ell}$, where $\ell \stackrel{\text { def }}{=} \operatorname{dim} V$. Throughout the paper we will call such $u_{1}, \ldots, u_{\ell}$ homogeneous generators or, collectively, a homogeneous generating set of $S(V)^{W}$. The homogeneous generators $u_{1}, \ldots, u_{\ell}$ are not unique, but their degrees $d_{1}, \ldots, d_{\ell}$ (which we traditionally list in the increasing order) are uniquely defined for each group $W$; they are called the exponents of the group. In particular, $d_{1}=2$ iff $V^{W}=\{0\}$; the largest exponent $h \stackrel{\text { def }}{=} d_{\ell}$ is called the Coxeter number of $W$. The monomials $u^{a} \stackrel{\text { def }}{=} u_{1}^{a_{1}} \cdots u_{\ell}^{a_{\ell}}$ where $a_{1}, \ldots, a_{\ell} \in \mathbb{Z}_{\geq 0}$ form an additive basis in $S(V)^{W}$.

Let $\prec$ be the inverse lexicographic order on $\mathbb{Z}_{\geq 0}^{\ell}$ : for $a, a^{\prime} \in \mathbb{Z}_{\geq 0}^{\ell}$ we write $a^{\prime} \prec a$ if the last non-zero coordinate of the vector $a-a^{\prime}$ is positive. The following is our first result asserting the existence and uniqueness of canonical invariants:

Theorem 1.1 (Canonical invariants). Suppose that the degrees $d_{1}, \ldots, d_{\ell}$ are all distinct. Then for each $a=\left(a_{1}, \ldots, a_{\ell}\right) \in \mathbb{Z}_{\geq 0}^{\ell}$ there exists a homogeneous element $b_{a}=b_{a}^{(c)} \in S_{c}(V)^{W}=$ $\mathbb{C}(c) \otimes S(V)^{W}$ unique up to multiplication by a complex constant and such that for any homogeneous generating set $u_{1}, \ldots, u_{\ell}$ of $S(V)^{W}$ one has:

1. $b_{a} \in \mathbb{C}^{\times} \cdot u^{a}+\sum_{a^{\prime} \prec a} \mathbb{C}(c) \cdot u^{a^{\prime}}$;

2. $\left(u^{a^{\prime}}, b_{a}\right)_{c}=0$ whenever $a^{\prime} \prec a$.

We will prove Theorem 1.1 in Section 3.1. We will refer to each element $b_{a}$ as a canonical $W$-invariant in $S_{c}(V)^{W}$ and to the set $\mathbf{B}=\left\{b_{a} \mid a \in \mathbb{Z}_{\geq 0}^{\ell}\right\}$, as the canonical basis of $S_{c}(V)^{W}$. By the construction, the canonical basis $\mathbf{B}$ is orthogonal with respect to the form (1.1).

Remark 1.1. We can extend the theorem to the case when $d_{k}=d_{k+1}$ for some $k$. If $V$ is irreducible, then this happens only when $W$ is of type $D_{\ell}$ with even $\ell$ and $k=\ell / 2$. In this case $V=\sum_{i=1}^{\ell} \mathbb{C} \cdot x_{i}$, the positive roots are of the form $x_{i} \pm x_{j}$, and let $\sigma: V \rightarrow V$ be the involution given by $\sigma\left(x_{i}\right)=\left\{\begin{array}{ll}x_{i} & \text { if } i<\ell \\ -x_{\ell} & \text { if } i=\ell\end{array}\right.$, i.e., $\sigma$ is acting on roots as the symmetry of the Dynkin diagram. Then Theorem 1.1 holds verbatim for any choice of homogeneous generators $u_{1}, \ldots, u_{\ell}$ of $S(V)^{W}$ such that $\sigma\left(u_{\ell / 2}\right)=-u_{\ell / 2}$ (i.e., $u_{\ell / 2} \in \mathbb{C} \cdot x_{1} \cdots x_{\ell}$ ) and $\sigma\left(u_{j}\right)=u_{j}$ for all $j \neq \ell / 2$.

An equality $d_{k}=d_{k+1}$ can also happen when $V$ is reducible, i.e., $V=V_{1} \oplus V_{2}, W=W_{1} \times W_{2}$ and each $W_{i}$ is a reflection group of $V_{i}$. This case can be handled by induction because $S(V)^{W}=$ $S\left(V_{1}\right)^{W_{1}} \otimes S\left(V_{2}\right)^{W_{2}}$. 
Remark 1.2. Theorem 1.1 generalizes to all complex reflection groups if one replaces the symmetric bilinear form on $V$ with the Hermitian one that canonically extends the $W$-invariant Hermitian form on $S_{c}(V)$ (provided that $c\left(s^{-1}\right)=\overline{c(s)}$ for all complex reflections $s$ ). The case of equal degrees $d_{k}$ can be treated along the lines of Remark 1.1. More precisely, the phenomenon $d_{k}=d_{k+1}$ occurs only for the following irreducible complex reflection groups (see e.g., $\left.[7,5]\right)$ :

1. The series $G(m, p, \ell)$ with $\ell \geq 2, p|\ell, p| m$, and $d_{k}=d_{k+1}=m \ell / p, k=\ell / p$.

2. The exceptional groups $G_{7}, G_{11}, G_{19}$ of rank $\ell=2$ with $d_{1}=d_{2}=12,24,60$, respectively.

In the case 1 , similarly to Remark 1.1, one has $V=\sum_{i=1}^{\ell} \mathbb{C} \cdot x_{i}, \sigma: V \rightarrow V$ is the automorphism given by $\sigma\left(x_{i}\right)=\left\{\begin{array}{ll}x_{i} & \text { if } i<\ell \\ \zeta x_{\ell} & \text { if } i=\ell\end{array}\right.$, where $\zeta$ is an $m$-th primitive root of unity. Then Theorem 1.1 holds verbatim for any choice of homogeneous generators $u_{1}, \ldots, u_{\ell}$ of $S(V)^{W}$ such that $\sigma\left(u_{k}\right)=\zeta^{m / p} u_{k}$ (i.e., $u_{k} \in \mathbb{C} \cdot\left(x_{1} \cdots x_{\ell}\right)^{m / p}$ ) and $\sigma\left(u_{i}\right)=u_{i}$ for all $i \neq k$.

In the case 2 one can use various embeddings of rank 2 complex reflection groups (see e.g., [7, Section 3]) to acquire canonical invariants. For instance, $G_{5}$ is a normal subgroup of index 2 in $G_{7}$ and $G_{5}$ has degrees $(6,12)$, which implies that if $\left\{b_{\left(a_{1}, a_{2}\right)}^{(c)} \mid a_{1}, a_{2} \in \mathbb{Z}_{\geq 0}\right\}$ is the canonical basis for $S(V)^{G_{5}}$, then the set $\left\{b_{\left(2 a_{1}, a_{2}\right)}^{(c)} \mid a_{1}, a_{2} \in \mathbb{Z}_{\geq 0}\right\}$ is a (canonical) basis of $S(V)^{G_{7}}$.

Therefore, the invariants $b_{a}^{(c)}$ make sense for all complex reflection groups.

Assume that $V^{W}=\{0\}$, i.e. $d_{1}=2$, and denote by $L$ the Dunkl Laplacian $\nabla_{e_{2}}: S_{c}(V) \rightarrow$ $S_{c}(V)$, where $e_{2}$ is the only (up to a scalar multiple) quadratic $W$-invariant in $S(V)$. Clearly, the restriction of $L$ to $S_{c}(V)^{W}$ is a well-defined linear operator $S_{c}(V)^{W} \rightarrow S_{c}(V)^{W}$.

Proposition 1.1. Assume that $V^{W}=\{0\}$. Then

(a) For each $r \geq 0$ the span of all $b_{\left(a_{1}, a_{2}, \ldots, a_{\ell}\right)}$ with $a_{1} \leq r$ is the kernel of the operator $\left.L^{r+1}\right|_{S_{c}(V)^{W}}$.

(b) For each $a=\left(a_{1}, \ldots, a_{\ell}\right)$ we have:

$$
b_{a}=e_{2}^{a_{1}} b_{\left(0, a_{2}, \ldots, a_{\ell}\right)}
$$

(in particular, $\left.b_{\left(a_{1}, 0, \ldots, 0\right)}=e_{2}^{a_{1}}\right)$.

See Section 3.2 for the proof.

The elements $b_{\left(0, a_{2}, \ldots, a_{\ell}\right)}$ of the canonical basis are more elusive, however we compute them completely when $W$ is a dihedral group.

Theorem 1.2. Let $W=I_{2}(m)$ be the dihedral group of order $2 m, V=\mathbb{C}^{2}$.

(a) If $c\left(s_{1}\right)=c\left(s_{2}\right)=c$, then the generating function of all $b_{(0, k)}$ is given by

$$
\sum_{k \geq 0}\left(\begin{array}{l}
c \\
k
\end{array}\right) b_{(0, k)} t^{k}=\left(1+e_{m} t+e_{2}^{m} t^{2}\right)^{c}
$$

where $e_{2}$ and $e_{m}$ are elementary $W$-invariants (of degrees 2 and $m$ respectively).

(b) If $m$ is even and $c\left(s_{1}\right) \neq c\left(s_{2}\right)$, then (using the notation $C \stackrel{\text { def }}{=} c\left(s_{1}\right)+c\left(s_{2}\right), \delta \stackrel{\text { def }}{=} c\left(s_{2}\right)-c\left(s_{1}\right)$, $\left.e_{m}^{\prime}=\frac{1}{4} e_{m}-\frac{1}{2} e_{2}^{m / 2}\right)$ we have:

$$
\sum_{k \geq 0} \frac{\Gamma\left(\frac{C-\delta-1}{2}\right) \Gamma(2 k-C)}{\Gamma\left(k-\frac{C+\delta-1}{2}\right)} b_{(0, k)} t^{k}=\int_{0}^{1}\left(1-\tau+t \tau\left(e_{2}^{m / 2}+\tau e_{m}^{\prime}\right)\right)^{\frac{C-\delta-1}{2}} \tau^{-C-1} d \tau
$$


We will prove the theorem in Section 3.5 by explicitly reducing the Dunkl Laplacians to the Jacobi operators. In fact, it is easy to see that the formula (1.2) is equivalent to:

$$
b_{(0, k)}=\frac{k ! e_{2}^{m k / 2}}{4^{k}\left(\begin{array}{c}
2 k-C+1 \\
k
\end{array}\right)} P_{k}^{\left(-\frac{C+\delta+1}{2},-\frac{C-\delta+1}{2}\right)}\left(\frac{e_{m}}{2 e_{2}^{m / 2}}\right)
$$

where $P_{k}^{(a, b)}(y)$ is the $k$-th Jacobi polynomial (see e.g. [1, Section 6.3] or formula (3.7) below). This and other of our arguments bear some similarity with methods of the seminal papers [8] and [11] where Jacobi polynomials were first studied in the context of Dunkl operators.

Returning to the general case, note that $\operatorname{deg} b_{a}=\sum d_{k} a_{k}$. For each $d \in \mathbb{Z}_{\geq 0}$ such that $S(V)_{d}^{W} \neq\{0\}$ we set $e_{d}^{(c) \stackrel{\text { def }}{=}} b_{a_{\max }}$, where $a_{\max } \in \mathbb{Z}_{\geq 0}^{\ell}$ is maximal with respect to $\prec$ among all $a \in \mathbb{Z}_{\geq 0}^{\ell}$ such that $\sum d_{k} a_{k}=d$. By the construction, $\operatorname{deg} e_{d}^{(c)}=d$. The following result was essentially proved in our previous paper [2].

Theorem 1.3. Let the exponents $d_{1}<\cdots<d_{\ell}$ be pairwise distinct. Then

(a) The elements $e_{d_{1}}^{(c)}, \ldots, e_{d_{\ell}}^{(c)}$ generate the algebra $S_{c}(V)^{W}$.

(b) Each $e_{d_{k}}^{(c)}$ is determined (up to a multiple) by its homogeneity degree $d=d_{k}$ and the equation $\nabla_{P}\left(e_{d_{k}}^{(c)}\right)=0$ for any $W$-invariant polynomial $P \in S_{c}(V)^{W}$ such that $\operatorname{deg} P<d_{k}$.

(c) For each $k=1,2, \ldots$ there is a unique, up to a multiple, element $e_{k h}^{(c)} \in S_{c}(V)^{W}$ (where $h=d_{\ell}$ is the Coxeter number) of the homogeneity degree $d=k h$ satisfying the equation $\nabla_{P}\left(e_{k h}^{(c)}\right)=0$ for any $W$-invariant polynomial $P \in S(V)^{W}$ such that $\operatorname{deg} P<h$.

We will give a new proof of Theorem 1.3 in Section 3.2. The proof will rely on the construction of canonical invariants in Theorem 1.1.

Following [2], we refer to each $e_{d_{k}}^{(c)}$ as the canonical elementary $W$-invariant and each $e_{k h}^{(c)}$ as the canonical quasiharmonic $W$-invariant.

The elementary invariants for $c=0$ were, most apparently, defined by Dynkin (see e.g. [16]) and later explicitly computed by K. Iwasaki in [15]. We extend the results of [15] to all $c$ in Theorem 1.4 below.

We will also construct elementary invariants for $W=S_{n}, V=\mathbb{C}^{n}$ with the natural $S_{n}$-action. It is convenient to identify $S_{c}(V)$ with the algebra $\mathbb{C}(c)\left[x_{1}, \ldots, x_{n}\right]$ of polynomials in $n$ variables depending rationally on $c$. The degrees $d_{k}$ are here $d_{k}=k, k=1, \ldots, n$, so Theorem 1.1 and Theorem 1.3 are applicable.

To give the explicit formula for the invariants $e_{k}^{(c)}$ define polynomials $\mu_{k}^{(c)} \in \mathbb{C}(c)\left[x_{1}, \ldots, x_{n}\right]$, $k=2, \ldots, n$, by

$$
\mu_{k}^{(c)}=\sum_{s=1}^{k}(-1)^{s} x_{s}\left(\Delta\left(\nabla_{x_{1}}, \ldots, \widehat{\nabla_{x_{s}}}, \ldots, \nabla_{x_{k}}\right)\right) \Delta\left(x_{1}, \ldots, x_{k}\right)
$$

where $\Delta\left(z_{1}, \ldots, z_{r}\right)=\prod_{1 \leq i<j \leq r}\left(z_{i}-z_{j}\right)$ is the Vandermonde determinant. Clearly, $\mu_{k}^{(c)} \in$ $\mathbb{C}(c)\left[x_{1}, \ldots, x_{n}\right]^{S_{k} \times S_{n-k}}$.

Theorem 1.4. For all $2 \leq k \leq n$ there exists $\alpha_{k, n} \in \mathbb{C}(c)^{\times}$such that the $k$-th elementary canonical invariant $e_{k}^{(c)} \in \mathbb{C}(c)\left[x_{1}, \ldots, x_{n}\right]^{S_{n}}$ is given by:

$$
e_{k}^{(c)}=\alpha_{k, n}(c) \sum_{w \in S_{n} /\left(S_{k} \times S_{n-k}\right)} w\left(\mu_{k}^{(c)}\right) .
$$


We prove Theorem 1.4 in Section 3.3. Our proof (as well as the formula (1.3)) is very similar to the one by K. Iwasaki who (using $\partial_{p}$ instead of Dunkl operators $\nabla_{p}$ ) computed $e_{k}^{(0)}$ in [15]. Following his argument, one can construct the elementary canonical invariants $e_{d_{k}}^{(c)}$ for other classical groups as well.

Note that the formula (1.3) resembles the polynomial expansion of the elementary symmetric polynomial $e_{k}=e_{k}\left(x_{1}, \ldots, x_{n}\right)$ :

$$
e_{k}=\sum_{w \in S_{n} /\left(S_{k} \times S_{n-k}\right)} w\left(x_{1} \cdots x_{k}\right)=\sum_{1 \leq j_{1}<\cdots<j_{k} \leq n} x_{j_{1}} \cdots x_{j_{k}} .
$$

The following main result demonstrates that this observation is not a mere coincidence.

Theorem 1.5. Let $W=S_{n}$. Then for all $k=2, \ldots, n$ the elementary canonical invariants $e_{k}^{(c)}$ have no poles at the singular value $c=1 / n$, and

$$
\lim _{c \rightarrow 1 / n} e_{k}^{(c)}=e_{k}\left(x_{1}-\frac{e_{1}(x)}{n}, \ldots, x_{n}-\frac{e_{1}(x)}{n}\right) .
$$

This result allows to introduce the elementary invariant polynomials for other reflection groups via $e_{d_{k}}=\lim _{c \rightarrow 1 / h} e_{d_{k}}^{(c)}$, where $h$ is the Coxeter number.

We will prove Theorem 1.5 in Section 3.4 by analyzing the behaviour of the form (1.1) near $c=1 / n$. Note, however, that we could not derive the theorem directly from the explicit formula (1.3).

Example 1.1. Denote $\bar{e}_{k}(x) \stackrel{\text { def }}{=} e_{k}\left(x_{1}-\frac{e_{1}(x)}{n}, \ldots, x_{n}-\frac{e_{1}(x)}{n}\right)$. It is easy to see that $e_{1}^{(c)}=0$, $e_{k}^{(c)}=\bar{e}_{k}$ for $k=2,3$. Direct computations for all $n$ using (1.3) show that

$$
\begin{aligned}
& e_{4}^{(c)}=\frac{(n-2)(n-3)}{2 n} \frac{1-n c}{\left(n^{2}-n\right) c-n-1} \bar{e}_{2}^{2}+\bar{e}_{4}, \\
& e_{5}^{(c)}=\frac{(n-3)(n-4)}{n} \frac{1-n c}{\left(n^{2}-n\right) c-n-5} \bar{e}_{2} \bar{e}_{3}+\bar{e}_{5},
\end{aligned}
$$

thus confirming Theorem 1.5.

Remark 1.3. The definition of the canonical invariants $b_{a}$ and some later formulas involving them (e.g. (2.2) and (2.3)) suggest, for $W=S_{n}$, a close relation between canonical invariants $b_{a}$ and Jack polynomials $J_{\lambda}^{(\alpha)}$ (see e.g. [19] for definition). Direct computations show, though, that these polynomials are not the same. [17, equation (7)] shows, in particular, that the expression of $J_{\lambda}^{(\alpha)}$ via elementary symmetric polynomials $e_{i}$ does not depend on $n$; for instance, $J_{(11 \ldots 1)}^{(\alpha)}=e_{k}$ for all $n$ and $k$ (the partition contains $k$ units). Formulas for $b_{a}$, on the contrary, contain $n$ explicitly (see e.g. (1.4)). So, the relation between $b_{a}$ and Jack polynomials is yet to be clarified.

\section{The Dunkl Laplacian and the scalar product}

Throughout the section we assume that $2=d_{1}<\cdots<d_{\ell}=h$ and denote

$$
e_{2}=\sum_{i=1}^{\ell} x_{i}^{2},
$$


where $x_{1}, \ldots, x_{\ell}$ is any orthonormal basis in the real space $V_{\mathbb{R}}$. Obviously, $e_{2}$ is a unique (up to a scalar multiple) quadratic $W$-invariant in $S^{2}(V)$. The operator $L=\nabla_{e_{2}}=\sum_{i} \nabla_{x_{i}}^{2}$, called the Dunkl Laplacian, is independent of the choice of the basis $x_{i}$; it equals the ordinary Laplacian if $c=0$.

The operator $L$ plays a key role in the theory of Dunkl operators for $W$. As the following result shows, an action of any Dunkl operator can be expressed via $L$ :

Lemma 2.1 ([3, equation (1.9)]). For any $p \in S^{d}(V)$ one has

$$
\nabla_{p}=\frac{1}{d !}(\operatorname{ad} L)^{d}(p)=\sum_{k=0}^{d} \frac{(-1)^{k}}{k !(d-k) !} L^{d-k} \cdot p \cdot L^{k}
$$

where $p$ in the right-hand side means the operator of multiplication by $p$.

Denote by $\mathcal{E}: S_{c}(V) \rightarrow S_{c}(V)$ the Euler vector field given by $\mathcal{E}(f)=N f$ for any $f \in S_{c}^{N}(V)$. Also denote $h_{c} \stackrel{\text { def }}{=} \frac{2}{\ell} \sum_{s \in S} c(s)$ (in particular, if all $c(s)$ are equal to a single $c$, then $h_{c}=h c$ ).

Proposition 2.1 ([14]). The operator $E$ of multiplication by $e_{2}$, Dunkl Laplacian L, and the operator $H \stackrel{\text { def }}{=} 2 \ell\left(1-h_{c}\right)+4 \mathcal{E}$ form a representation of $\mathfrak{s l}_{2}$, that is,

$$
[E, L]=H, \quad[H, E]=2 E, \quad[H, L]=-2 L .
$$

In particular,

$$
\left[L, E^{k}\right]=4 k E^{k-1}\left(\ell\left(1-h_{c}\right) / 2+k-1+\mathcal{E}\right)
$$

for all $k \geq 0$.

Denote $U_{d}=U_{d}^{(c)} \stackrel{\text { def }}{=} \operatorname{Ker} L \cap S_{c}^{d}(V)=\left\{f \in S_{c}^{d}(V) \mid L(f)=0\right\}$.

Lemma 2.2. One has

$$
S_{c}(V)=\bigoplus_{k, d \in \mathbb{Z}_{\geq 0}} e_{2}^{k} \cdot U_{d}^{(c)}
$$

where the direct summands are orthogonal with respect to $(\cdot, \cdot)_{c}$. In particular, the restriction of $(\cdot, \cdot)_{c}$ to each $e_{2}^{k} \cdot U_{d}$ is nondegenerate.

Proof. First, note that $S_{c}(V)$ is an $\mathfrak{s l}_{2}$-module, locally finite with respect to $L$, and $\oplus_{d \geq 0} U_{d}^{(c)}$ is the highest weight space, so that decomposition (2.1) takes place.

Furthermore, note that the operator $H$ from Proposition 2.1 is scalar on the space of polynomials of any given degree and therefore self-adjoint; the operators $L$ and $E$ are adjoint to one another with respect to $(\cdot, \cdot)_{c}$. Therefore, for $k_{1} \leq k_{2}, d_{1}, d_{2} \geq 0$ one has

$$
\begin{aligned}
\left(e_{2}^{k_{1}} \cdot U_{d_{1}}^{(c)}, e_{2}^{k_{2}} \cdot U_{d_{2}}^{(c)}\right)_{c} & =\left(E^{k_{1}}\left(U_{d_{1}}^{(c)}\right), E^{k_{2}}\left(U_{d_{2}}^{(c)}\right)\right)_{c}=\left(L^{k_{2}} E^{k_{1}}\left(U_{d_{1}}^{(c)}\right), U_{d_{2}}^{(c)}\right)_{c} \\
& =\delta_{k_{1}, k_{2}} \cdot\left(U_{d_{1}}^{(c)}, U_{d_{2}}^{(c)}\right)_{c}=\delta_{k_{1}, k_{2}} \delta_{d_{1}, d_{2}} \cdot \mathbb{C}(c) .
\end{aligned}
$$

This proves the orthogonality of the decomposition. In particular, this implies that the restriction of the nondegenerate form $(\cdot, \cdot)_{c}$ to each $e_{2}^{k} \cdot U_{d}$ is nondegenerate. The lemma is proved.

Using this, we compute the form $(\cdot, \cdot)_{c}$ as follows. Denote by $\varphi_{c}$ a (unique) linear function $S_{c}(V) \rightarrow \mathbb{C}(c)$ such that:

- $\varphi_{c}\left(f e_{2}\right)=\varphi_{c}(f)$ for all $f \in S_{c}(V)$;

- $\varphi_{c}(f)=[f]_{0}$ for all $f \in \operatorname{Ker} L$, where $[\cdot]_{0}: S_{c}(V) \rightarrow \mathbb{C}(c)$ is the projection defined in (1.1). 
Proposition 2.2. We have:

(a) For $f \in e_{2}^{k} U_{d}^{(c)}, g \in S_{c}^{d+2 k}(V)$ one has

$$
(f, g)_{c}=\varphi_{c}(f g) \cdot 4^{d+k} k ! \prod_{r=0}^{d+k-1}\left(\ell\left(1-h_{c}\right) / 2+r\right) .
$$

(b) If $c: S / W \rightarrow \mathbb{R}_{<1 / 2}$, then the restriction of $\varphi_{c}$ to $S\left(V_{\mathbb{R}}\right)$ is given by:

$$
\varphi_{c}(f)=\frac{\int_{\Omega^{\ell-1}} f(x) \cdot \prod_{s \in S}\left|\alpha_{s}(x)\right|^{-2 c(s)} d x}{\int_{\Omega^{\ell-1}} \prod_{s \in S}\left|\alpha_{s}(x)\right|^{-2 c(s)} d x},
$$

where $\alpha_{s} \in V_{\mathbb{R}}$ is a coroot of a reflection $s \in S, \Omega^{\ell-1}=\left\{x \in V_{\mathbb{R}} \mid e_{2}(x)=1\right\}$ is the unit sphere in $V_{\mathbb{R}}$, and an element $f \in S(V)$ is identified with a polynomial on $V$.

Proof. Assume first that the function $c$ takes only negative real values and define $\varphi_{c}$ by equation (2.3). Now if $k=0$ and $f, g \in U_{d}^{(c)}$, then the result follows from [12, Theorem 5.2.4].

By definition, $\varphi_{c}\left(e_{2} f\right)=\varphi_{c}(f)$ for any $f$. Now if $f=e_{2}^{k} \tilde{f}, g=e_{2}^{k^{\prime}} \tilde{g}$ where $\tilde{f} \in U_{d}^{(c)}, \tilde{g} \in U_{d^{\prime}}^{(c)}$ with $d \neq d^{\prime}$ then $(\tilde{f}, \tilde{g})_{c}=0$, hence $\varphi_{c}(\tilde{f} \tilde{g})=0$ and therefore $\varphi_{c}(f g)=0$. So taking $f \in e_{2}^{k} U_{d}^{(c)}$ and $g=\sum_{r} e_{2}^{r} \tilde{g}_{r} \in S_{c}^{d+2 k}(V)$, where $\tilde{g}_{r} \in U_{d+2 k-2 r}$, we see that

$$
\varphi_{c}(f g)=\varphi_{c}\left(\tilde{f} \cdot \tilde{g}_{k}\right) \text {. }
$$

On the other hand, decomposition (2.1) guarantees that $(f, g)_{c}=\left(\tilde{f}, \tilde{g}_{k}\right)_{c}$. Therefore, to verify (2.2) for any $k$ it suffices to take $g=e_{2}^{k} \tilde{g}$ for $g \in U_{d}^{(c)}$.

Assume that $k>0$. Then Proposition 2.1 implies that

$$
\begin{aligned}
\left(e_{2}^{k} \tilde{f}, e_{2}^{k} \tilde{g}\right)_{c} & =\left(E^{k}(\tilde{f}), E^{k}(\tilde{g})\right)_{c}=\left(E^{k-1}(\tilde{f}), L E^{k}(\tilde{g})\right)_{c}=\left(E^{k-1}(\tilde{f}),\left[L, E^{k}\right](\tilde{g})\right)_{c} \\
& \left.=\left(E^{k-1}(\tilde{f}), 4 k E^{k-1}\left(\ell\left(1-h_{c}\right) / 2+k-1+\mathcal{E}\right) \tilde{g}\right)\right)_{c} \\
& =4 k\left(\ell\left(1-h_{c}\right) / 2+k-1+d\right)\left(e_{2}^{k-1}(\tilde{f}), e_{2}^{k-1} \tilde{g}\right)_{c} .
\end{aligned}
$$

Therefore, by induction on $k$,

$$
\left(e_{2}^{k} \tilde{f}, e_{2}^{k} \tilde{g}\right)_{c}=(\tilde{f}, \tilde{g})_{c} \cdot \prod_{r=1}^{k} 4 r\left(\ell\left(1-h_{c}\right) / 2+r-1+d\right),
$$

which finishes the proof for $c$ negative real. Now (2.2) implies that for $c$ negative real the value $(f, g)_{c}$ depends only on the product $f g$ (provided $d$ and $k$ are fixed). Since $(f, g)_{c}$ is a rational function of the values of $c$, this holds true for all $c$ as well - so, one can use (2.2) to define $\varphi_{c}$ in the general case.

The following is the main result of the section. Denote by $S(V)_{+}$the kernel of the constant term projection $u \rightarrow[u]_{0}$, see (1.1). Define a symmetric bilinear form $\Phi_{c}: S(V)_{+} \times S(V)_{+} \rightarrow$ $\mathbb{C}(c)$ as $\Phi_{c}(u, v) \stackrel{\text { def }}{=}(u, v)_{c} /\left(1-h_{c}\right)$. This form extends naturally to $\mathbb{C}[c] \otimes S(V)$. Define now the form $\bar{\Phi}_{c}$ on $\mathbb{C}[c] /\left(1-h_{c}\right) \otimes S(V)$ taking values in $\mathbb{C}[c] /\left(1-h_{c}\right)$ by

$$
\bar{\Phi}_{c}(u, v)=\pi\left(\Phi_{c}(\tilde{u}, \tilde{v})\right),
$$

where $\pi: \mathbb{C}[c] \rightarrow \mathbb{C}[c] /\left(1-h_{c}\right)$ is the canonical projection and $\tilde{u}, \tilde{v} \in S_{c}(V)$ are any elements such that $u=\pi(\tilde{u}), v=\pi(\tilde{v})$. 


\section{Theorem 2.1.}

(a) The form $\Phi_{c}$ takes its values in $\mathbb{C}[c]$.

(b) $\bar{\Phi}_{c}(u, u) \neq 0$ for any non-zero element $u \in \mathbb{R}[c] /\left(1-h_{c}\right) \otimes_{\mathbb{R}} S\left(V_{\mathbb{R}}\right)_{+}$.

(c) For any $U_{\mathbb{R}} \subset S\left(V_{\mathbb{R}}\right)_{+}$the restriction of $\bar{\Phi}_{c}$ to $\mathbb{C}[c] /\left(1-h_{c}\right) \otimes U_{\mathbb{R}}$ is nondegenerate.

Proof. Prove (a) by induction on the degree of $u$. Indeed, it follows from [4, Proposition 2.1] that for any $x, y \in V$ one has:

$$
(x, y)_{c}=\left(1-h_{c}\right)(x, y)_{0},
$$

where $(x, y)_{0}$ is the (complexified) $W$-invariant form on $V$. Therefore, $\left.\Phi_{c}\right|_{V \times V}=(\cdot, \cdot)_{0}$. Furthermore, assume that $\Phi_{c}(u, v) \in \mathbb{C}[c]$ for all $u, v \in S^{<d}(V)_{+}$. Then for any $u_{1} \in S^{d_{1}}(V)$, $u_{2} \in S^{d_{2}}(V), v \in S^{d}(V)$, where $d_{1}+d_{2}=d$, we have

$$
\Phi_{c}\left(u_{1} u_{2}, v\right)=\Phi_{c}\left(u_{2}, \nabla_{u_{1}}(v)\right) \in \Phi_{c}\left(u_{2}, \mathbb{C}[c] \otimes S^{d_{2}}(V)\right) \subset \mathbb{C}[c] .
$$

This proves $(a)$.

It is possible to prove $(b)$ now. Let $H_{0} \stackrel{\text { def }}{=}\left\{c \in \mathbb{C}^{S / W} \mid h_{c}=1\right\}$; it is an affine hyperplane in the affine space $\mathbb{A}^{S / W}$. Then $\mathbb{C}\left[H_{0}\right]=\mathbb{C}[c] /\left(1-h_{c}\right)$ and $\mathbb{R}\left[H_{0}\right]=\mathbb{R}[c] /\left(1-h_{c}\right)$ are integral domains (if $|S / W|=1$ then $H_{0}$ is a point $c=1 / h$ and $\mathbb{R}\left[H_{0}\right]=\mathbb{R}$ ).

Let $\mathcal{A} \subset \mathbb{R}\left(\mathbb{A}^{S / W}\right)$ be the algebra of all real-valued rational functions on the affine space $\mathbb{A}^{S / W}$ regular at $H_{0}$. This algebra is local with the maximal ideal $\mathfrak{m}=\left(1-h_{c}\right)$, and $\mathcal{A} / \mathfrak{m}$ is isomorphic to $\mathbb{R}\left(H_{0}\right)$, the field of fractions of $H_{0}$. Finally, denote $S_{\mathcal{A}}\left(V_{\mathbb{R}}\right)_{+}=\mathcal{A} \otimes_{\mathbb{R}} S(V)_{+}$.

Proposition 2.3. The naturally extended $\mathcal{A}$-linear form $\Phi_{c}: S_{\mathcal{A}}\left(V_{\mathbb{R}}\right)_{+} \times S_{\mathcal{A}}\left(V_{\mathbb{R}}\right)_{+} \rightarrow \mathcal{A}$ satisfies:

$$
\text { if } \Phi_{c}(\tilde{u}, \tilde{u}) \in\left(1-h_{c}\right) \mathcal{A} \text { for some } \tilde{u} \in S_{\mathcal{A}}\left(V_{\mathbb{R}}\right) \text { then } \tilde{u} \in\left(1-h_{c}\right) S_{\mathcal{A}}\left(V_{\mathbb{R}}\right) \text {. }
$$

Proof. Clearly, the $\mathfrak{s l}_{2}$-action from Proposition 2.1 preserves both $\mathbb{R}[c] \otimes S\left(V_{\mathbb{R}}\right)$ and $S_{\mathcal{A}}\left(V_{\mathbb{R}}\right)$, so that the orthogonal decomposition $(2.1)$ is valid for $S_{\mathcal{A}}\left(V_{\mathbb{R}}\right) \subset S_{c}(V)$. Therefore, it suffices to verify (2.4) only for $\tilde{u} \in e_{2}^{k} \tilde{U}_{d}$, where

$$
\tilde{U}_{d}=U_{d}^{(c)} \cap S_{\mathcal{A}}\left(V_{\mathbb{R}}\right)=\left\{\tilde{v} \in S_{\mathcal{A}}\left(V_{\mathbb{R}}\right) \mid L(\tilde{v})=0\right\} .
$$

For every such $\tilde{u}$ it follows from (2.2) that

$$
\Phi_{c}(\tilde{u}, \tilde{u})=\varphi_{c}\left(\tilde{u}^{2}\right) \cdot 2 \cdot 4^{d+k-1} k ! \prod_{r=1}^{d+k-1}\left(\ell\left(1-h_{c}\right) / 2+r\right) .
$$

Since the product in the right-hand side is not divisible by $\left(1-h_{c}\right)$, we see that $\varphi_{c}\left(\tilde{u}^{2}\right) \in \mathcal{A}$ for all $\tilde{u} \in S_{\mathcal{A}}\left(V_{\mathbb{R}}\right)$. Implication (2.4) is now equivalent to the following one:

$$
\text { if } \varphi_{c}\left(\tilde{u}^{2}\right) \in\left(1-h_{c}\right) \mathcal{A} \text { for some } \tilde{u} \in S_{\mathcal{A}}\left(V_{\mathbb{R}}\right) \text { then } \tilde{u} \in\left(1-h_{c}\right) S_{\mathcal{A}}\left(V_{\mathbb{R}}\right) \text {. }
$$

In (2.6), if $h=2$, i.e., $W=S_{2}$, we have nothing to prove. Assume that $h>2$ and let $\tilde{H}_{0}$ be the set of all $c_{0}: S / W \rightarrow \mathbb{R}_{<1 / 2}$ such that $1-h_{c_{0}}=0$ and $\tilde{u}$ has no poles at $c_{0}$. By the very design, $\tilde{H}_{0}$ is a non-empty open subset of the real hyperplane $H_{0}(\mathbb{R}) \subset \mathbb{R}^{S / W}$. Indeed, we can write $h_{c}=h_{1} c_{1}+\cdots+h_{k} c_{k}$, where $k=|S / W|$ and $c_{1}, \ldots, c_{k}$ are standard coordinates on $\mathbb{A}^{S / W}=\mathbb{A}^{k}$, all $h_{i} \in \mathbb{Q}_{>0}$ and $h_{1}+\cdots+h_{k}=h$. The intersection $H_{0}^{\prime}=H_{0}(\mathbb{R}) \cap\left(\mathbb{R}_{<1 / 2}\right)^{k}$ contains the point $c=(1 / h, \ldots, 1 / h)$, hence $H_{0}^{\prime}$ is non-empty and open in $H_{0}(\mathbb{R})$. The set $\tilde{H}_{0}$ is obtained from $H_{0}^{\prime}$ by removing poles of $\tilde{u} \in \mathcal{A} \otimes S\left(V_{\mathbb{R}}\right)$; so, $\tilde{H}_{0} \subset H_{0}(\mathbb{R})$ is open and non-empty 
as well. On the other hand, for each $c_{0} \in \tilde{H}_{0}$ the specialization $\tilde{u}_{c_{0}} \in S\left(V_{\mathbb{R}}\right)$ of $\tilde{u}$ is well-defined. Therefore, our choice of $\tilde{u}$ and $c_{0}$ implies that

$$
\varphi_{c_{0}}\left(\tilde{u}_{c_{0}}^{2}\right)=0
$$

But the integral presentation (2.3) of $\varphi_{c_{0}}$ guarantees that $\varphi_{c_{0}}\left(f^{2}\right)>0$ for all nonzero polynomials $f \in S\left(V_{\mathbb{R}}\right)$. Hence, $\tilde{u}_{c_{0}}=0$, for all $c_{0} \in \tilde{H}_{0}$. If $|S / W|=1$, i.e., $H_{0}$ is a single point $c=1 / h$, then, clearly, $c_{0}=1 / h$ and $\tilde{u}_{1 / h}=0$ implies that $\tilde{u} \in(1-h c) S_{\mathcal{A}}\left(V_{\mathbb{R}}\right)$. Otherwise, if $H_{0}$ is at least an affine line, the set $\tilde{H}_{0}$ is infinite and is a set of regular points for a rational function $c_{0} \mapsto \tilde{u}_{c_{0}}$. Therefore, $\tilde{u}_{c_{0}}=0$ for all $c_{0} \in H_{0}(\mathbb{R})$ and hence $\tilde{u} \in\left(1-h_{c}\right) S_{\mathcal{A}}\left(V_{\mathbb{R}}\right)$ as well, proving implications (2.6) and (2.4). The proposition is proved.

Part $(b)$ of the theorem immediately follows from Proposition 2.3.

To prove $(c)$ we need the following obvious result:

Lemma 2.3. Let $\bar{\Phi}: U_{0} \times U_{0} \rightarrow \mathbb{k}$ be a non-degenerate symmetric bilinear form on a $\mathbb{k}$-vector space $U_{0}$. Then for any field $\mathbb{F}$ containing $\mathbb{k}$ the extension of $\Phi$ to $U=\mathbb{F} \otimes_{\mathbb{k}} U_{0}$ is a non-degenerate $\mathbb{F}$-bilinear form $U \times U \rightarrow \mathbb{F}$.

We will use the lemma with $\mathbb{k}$ being the field of fractions of the integral domain $\mathbb{R}[c] /\left(1-h_{c}\right)$, $\mathbb{F}$ - the field of fractions of $\mathbb{C}[c] /\left(1-h_{c}\right), U_{0}=\mathbb{k} \otimes U_{\mathbb{R}}$, and $\Phi=\bar{\Phi}_{c}$. It follows from part $(b)$ of the theorem that the restriction of $\bar{\Phi}_{c}$ to $U_{0}$ is non-degenerate. Hence Lemma 2.3 guarantees the same for $U=\mathbb{F} \otimes U_{0}$. This proves part $(c)$ of the theorem.

Remark 2.1. The proof of Theorem $2.1(b)$ also implies unitarity of $S\left(V_{\mathbb{R}}\right)_{+}$as a module over the rational Cherednik algebra $H_{c_{0}}(W)$ for all $c_{0} \in \mathbb{R}_{\leq 0}^{S / W}$ and for small $c_{0} \in \mathbb{R}_{>0}^{S / W}$. This agrees with the results of the recent paper [13], where the unitary representations of $H_{c}(W)$ were studied.

\section{Canonical basis and proofs of main results}

\subsection{Proof of Theorem 1.1}

Fix a homogeneous generating set $\left\{u_{1}, \ldots, u_{\ell}\right\}$ of $S(V)^{W}$ and take the basis $u^{a}, a \in \mathbb{Z}_{\geq 0}^{\ell}$, in $S(V)$ with the inverse lexicographic order. Define the $\mathbb{C}$-subspaces $S_{c}(V)_{\prec a}^{W}$ and $S_{c}(V)_{\preceq a}^{W}$ of $S_{c}(V)^{W}$ by

$$
S_{c}(V) \underset{\prec a}{W}=\sum_{a^{\prime} \prec a} \mathbb{C}(c) u^{a^{\prime}}, \quad S_{c}(V)_{\preceq a}^{W}=\mathbb{C} u^{a}+S(V)_{\prec a}^{W} .
$$

(clearly, $S_{c}(V) \underset{\prec a}{W} \subset S_{c}(V) \underset{\preceq}{W}$ ). Note first that for each $a \in \mathbb{Z}_{\geq 0}^{\ell}$ the spaces $S_{c}(V) \underset{\prec a}{W}, S_{c}(V) \underset{\preceq a}{W}$ do not depend on the choice of generators $u_{1}, \ldots, u_{\ell}$ of $S(V)^{W}$. Indeed, let $u_{1}^{\prime}, \ldots, u_{\ell}^{\prime}$ be another set of generators of $S(V)^{W}$. Since $d_{1}<d_{2}<\cdots<d_{\ell}$, one has $u_{i}^{\prime}=\alpha_{i} u_{i}+P_{i}\left(u_{1}, \ldots, u_{i-1}\right)$, where $\alpha_{i} \in \mathbb{C} \backslash\{0\}$ and $P_{i}$ is a polynomial of $i-1$ variables for $i=1,2, \ldots, \ell$.

We are going to define the canonical invariant $b_{a} \in S_{c}(V)_{\prec a}^{W}$ as the unique (up to a multiple) vector orthogonal to the subspace $S_{c}(V)_{\prec a}^{W}$. However, the uniqueness of such an element requires more arguments.

Proposition 3.1. Let $U$ be any subspace of $S\left(V_{\mathbb{R}}\right)$. Then:

(a) the restriction of the form (1.1) to $U_{c}=\mathbb{C}(c) \otimes U$ is a non-degenerate symmetric bilinear form on $U_{c}$;

(b) for any vector $u \in S\left(V_{\mathbb{R}}\right) \backslash U$ there is a unique (up to a complex multiple) element $b \in$ $\mathbb{C} \cdot u+U_{c}$ such that $\left(b, U_{c}\right)_{c}=0$. 
Remark 3.1. Statement $(a)$ of this proposition is essentially a Cherednik algebra version of the statements proved in [18, statements 5.1.20 and 5.1.21] for double affine Hecke algebras.

Proof. We need the following general fact. Let $\mathcal{A}$ be a unital commutative local ring with no zero-divisors, $\mathfrak{m}$ its maximal ideal, $\mathbb{k}=\mathcal{A} / \mathfrak{m}$ the residue field. In what follows we assume that $\mathbb{k} \subset \mathcal{A}$ so that the restriction of the canonical projection $\mathcal{A} \rightarrow \mathbb{k}$ to $\mathbb{k}$ is the identity homomorphism $\mathbb{k} \rightarrow \mathbb{k}$. Let $U$ be a vector space over $\mathbb{k}$ and let $\Phi: U \times U \rightarrow \mathcal{A}$ be a $\mathbb{k}$-bilinear symmetric form on $U$; denote by $\Phi_{0}: U \times U \rightarrow \mathbb{k}$ the residual form given by $\Phi_{0} \stackrel{\text { def }}{=} \pi \circ \Phi$, where $\pi: \mathcal{A} \rightarrow \mathbb{k}$ is the canonical quotient map.

Lemma 3.1. In the notation as above assume that:

1. $\Phi_{0}(u, u) \neq 0$ for all $u \in U \backslash\{0\}$.

2. There exists an increasing sequence of prime ideals

$$
\{0\}=\mathfrak{m}_{0} \subset \mathfrak{m}_{1} \subset \mathfrak{m}_{2} \subset \cdots \subset \mathfrak{m}_{k}=\mathfrak{m}
$$

in $\mathcal{A}$ such that $\mathfrak{m}_{i+1} / \mathfrak{m}_{i}$ is a principal ideal of $\mathcal{A} / \mathfrak{m}_{i}$ for $i=0,1, \ldots, k-1$.

Then the natural $\mathcal{A}$-bilinear extension of $\Phi$ to $U_{\mathcal{A}} \times U_{\mathcal{A}} \rightarrow \mathcal{A}$, where $U_{\mathcal{A}} \stackrel{\text { def }}{=} \mathcal{A} \otimes_{\mathbb{k}} U$, satisfies $\Phi(\tilde{u}, \tilde{u}) \neq 0$ for all $\tilde{u} \in U_{\mathbb{F}} \backslash\{0\}$.

Proof. We proceed by induction on $k$. For $k=0, \mathbf{m}=\{0\}, \mathbb{F}=\mathcal{A}=\mathbb{k}$, and we have nothing to prove. Assume that $k \geq 1$. Define the quotient $\operatorname{ring} \mathcal{A}^{\prime} \stackrel{\text { def }}{=} \mathcal{A} / \mathfrak{m}_{1}$, and $\mathfrak{m}_{i}^{\prime} \stackrel{\text { def }}{=} \mathfrak{m}_{i+1} / \mathfrak{m}_{1}$ in $\mathcal{A}^{\prime}$ for $i=0, \ldots, k-1$. Clearly, the $\operatorname{ring} \mathcal{A}^{\prime}$ and its ideals $\mathfrak{m}_{i}^{\prime}$ satisfy the assumptions of the lemma for $k-1$; therefore, the inductive hypothesis holds in the following form:

$$
\text { if } \Phi(\tilde{u}, \tilde{u}) \in \mathfrak{m}_{1} \text { for some } \tilde{u} \in \mathcal{A} \otimes U, \text { then } \tilde{u} \in \mathfrak{m}_{1} \otimes U \text {. }
$$

Since the ideal $\mathfrak{m}_{1}$ is principal, i.e., $\mathfrak{m}_{1}=c_{1} \mathcal{A}$, we can write each non-zero vector $\tilde{u} \in \mathfrak{m}_{1} \otimes U$ in the form $\tilde{u}=c_{1}^{\ell} \tilde{u}_{0}$, where $\tilde{u}_{0} \notin \mathfrak{m}_{1} \otimes U$. Therefore, the equation $\Phi(\tilde{u}, \tilde{u})=0$ is equivalent to $\Phi\left(\tilde{u}_{0}, \tilde{u}_{0}\right)=0$. However, applying the inductive hypothesis (3.2) to any $\tilde{u}_{0} \notin \mathfrak{m}_{1} \otimes U$ satisfying $\Phi\left(\tilde{u}_{0}, \tilde{u}_{0}\right)=0$, we obtain a contradiction. Therefore, $\Phi\left(\tilde{u}_{0}, \tilde{u}_{0}\right) \neq 0$ for all $u_{0} \notin \mathfrak{m}_{1} \otimes U$. Hence $\Phi(\tilde{u}, \tilde{u})=0$ for $\tilde{u} \in \mathcal{A} \otimes U$ if and only if $\tilde{u}=0$.

The lemma is proved.

We apply the lemma in the case when $\mathbb{F}=\mathbb{C}(c)=\mathbb{C}\left(c_{1}, \ldots, c_{k}\right)$ is the field of rational functions in the variables $c_{1}, \ldots, c_{k}$ (where $k=|S / W|$ is the number of conjugacy classes of reflections in $W), \mathcal{A} \subset \mathbb{F}$ is the local ring of all rational functions regular at $c=0$, and $\mathfrak{m}_{i}$ is the ideal of $\mathcal{A}$ generated by $c_{1}, \ldots, c_{i}$ for $i=0,1, \ldots, k$. Clearly, the ideals $\mathfrak{m}_{i}$ satisfy condition 2 of Lemma 3.1. Take $U$ to be any subspace of $S\left(V_{\mathbb{R}}\right)$ and let $\Phi: U \times U \rightarrow \mathcal{A} \subset \mathbb{R}(c)$ be the restriction of the form (1.1) to $U$. Since the specialization $\Phi_{0}$ of $\Phi$ at $c=0$ is a positive definite form on $U$, condition 1 of Lemma 3.1 holds as well.

Thus, Lemma 3.1 guarantees that for any $U \subset S\left(V_{\mathbb{R}}\right)$ each $\tilde{u} \in \mathbb{R}(c) \otimes U \backslash\{0\}$ satisfies $(\tilde{u}, \tilde{u})_{c} \neq 0$.

Therefore, the restriction of the form (1.1) to $\mathbb{R}(c) \otimes U$ is non-degenerate. By extending the coefficients from $\mathbb{R}(c)$ to $\mathbb{C}(c)$ this immediately proves assertion $(a)$ of Proposition 3.1.

To prove assertion $(b)$ denote $U_{c}^{\perp}=\left\{\tilde{u}^{\prime} \in \mathbb{C}(c) u+U_{c} \mid\left(\tilde{u}^{\prime}, U_{c}\right)_{c}=0\right\}$. Clearly, $U_{c}^{\perp} \neq 0$ and $\left(U_{c}^{\perp} \cap U_{c}, U_{c}\right)_{c}=0$; hence $U_{c}^{\perp} \cap U_{c}=0$ by assertion $(a)$. This implies that $\operatorname{dim} U_{c}^{\perp}=1$. Therefore, $U_{c}^{\perp}=\mathbb{C}(c) \cdot b$ for some $b \in u+U_{c}$. This completes the proof of Proposition 3.1. 
Now we are ready to finish the proof of Theorem 1.1. For each $a \in \mathbb{Z}_{\geq 0}^{\ell}$ denote by $S_{c}\left(V_{\mathbb{R}}\right)_{\prec a}=$ $S_{c}\left(V_{\mathbb{R}}\right) \cap S_{c}(V)_{\prec a}$ (see (3.1)) the real forms of $S(V)_{\prec a}$. Fix $u_{1}, \ldots, u_{\ell}$ to be a homogeneous generating set of $S\left(V_{\mathbb{R}}\right)^{W}$ so that (3.1) implies that $S_{c}\left(V_{\mathbb{R}}\right)_{\prec a}^{W}=\sum_{a^{\prime} \prec a} \mathbb{R}(c) u^{a^{\prime}}$ and $\mathbb{C} \otimes S_{c}\left(V_{\mathbb{R}}\right)_{\prec a}=$ $S_{c}(V)_{\prec a}$.

Therefore, Proposition 3.1 is applicable to this situation with $U=S_{c}\left(V_{\mathbb{R}}\right)_{\prec a}^{W}, u=u^{a}$, and there exists a unique (up to a complex multiple) element $b=b_{a} \in S_{c}(V)_{\preceq a}$ such that $\left(b_{a}, S_{c}(V)_{\prec a}^{W}\right)=0$ (in particular, $b_{a} \notin S_{c}(V)_{\prec a}^{W}$ ). In other words, $b_{a}$ satisfies both conditions of Theorem 1.1, and the theorem is proved.

\subsection{Proof of Proposition 1.1 and Theorem 1.3}

Proof of Proposition 1.1. To prove $(a)$, let $u_{1}\left(=e_{2}\right), u_{2}, \ldots, u_{\ell}$ be any homogeneous generating set of $S(V)^{W}$. Theorem 1.1 guarantees that for any $a=\left(a_{1}, \ldots, a_{\ell}\right), a^{\prime}=\left(a_{1}^{\prime}, \ldots, a_{\ell}^{\prime}\right) \in \mathbb{Z}_{\geq 0}^{\ell}$ with $a_{1}^{\prime}>a_{1}$ we have

$$
\left(u^{a^{\prime}}, b_{a}\right)_{c}=0 .
$$

Equivalently, taking into account that $\left(e_{2}^{r+1} u, b_{a}\right)_{c}=\left(u, L^{r+1}\left(b_{a}\right)\right)_{c}$ for all $r \geq 0$, we obtain $\left(S(V)^{W}, \nabla_{e_{2}}^{a_{1}+1}\left(b_{a}\right)\right)_{c}=0$. Since the form (1.1) is nondegenerate, we obtain

$$
L^{a_{1}+1}\left(b_{a}\right)=0
$$

for all $a \in \mathbb{Z}_{\geq 0}^{\ell}$. This proves that $\mathbf{B}_{r}=\left\{b_{a} \mid a_{1} \leq r\right\}$ is a (linearly independent) subset of the kernel of $\left.L^{r+1}\right|_{S_{c}(V)^{W}}$. On the other hand, since $e_{2}$ and $L$ form a representation of $\mathfrak{s l}_{2}$ by Proposition 2.1, we obtain isomorphisms of graded spaces:

$$
\begin{aligned}
& S_{c}(V)^{W} \cong \mathbb{C}(c)\left[e_{2}\right] \otimes \mathcal{K}, \\
& \text { Ker }\left.L^{n+1}\right|_{S_{c}(V)^{W}}=\left(\sum_{r=0}^{n} \mathbb{C}(c) \cdot e_{2}^{r}\right) \otimes \mathcal{K}
\end{aligned}
$$

where $\mathcal{K}$ is the kernel of $\left.L\right|_{S_{c}(V)^{W}}$. In particular, the Hilbert series of $\mathcal{K}$ is $\prod_{k=2}^{\ell} \frac{1}{1-t^{d_{k}}}$, so that

$$
\operatorname{dim}\left(\mathcal{K} \cap S_{c}^{d}(V)\right)=\left|\mathbf{B}_{0} \cap S_{c}^{d}(V)\right|,
$$

hence

$$
\operatorname{dim}\left(\left.\operatorname{Ker} L^{r+1}\right|_{S_{c}(V)^{W}} \cap S_{c}^{d}(V)\right)=\left|\mathbf{B}_{r} \cap S_{c}^{d}(V)\right|
$$

for all $r \geq 0$. This, together with the inclusion $\left.\mathbf{B}_{r} \subset \operatorname{Ker} L^{r+1}\right|_{S_{c}(V)^{W}}$, proves that $\mathbf{B}_{r}$ is a basis of Ker $\left.L^{n+1}\right|_{S_{c}(V)^{W}}$. Part $(a)$ is proved.

To prove $(b)$, denote

$$
\tilde{b}_{a}=e_{2}^{a_{1}} b_{\left(0, a_{2}, \ldots, a_{\ell}\right)}
$$

for each $a=\left(a_{1}, \ldots, a_{\ell}\right) \in \mathbb{Z}_{\geq 0}^{\ell}$. Since $\tilde{b}_{a}$ satisfies condition 1 of Theorem 1.1 , to prove that $b_{a}=\tilde{b}_{a}$ it suffices to verify that the elements $\tilde{b}_{a}$ satisfy condition 2 of the same theorem. This is equivalent to the elements $\tilde{b}_{a}$ being pairwise orthogonal, i.e., $\left(\tilde{b}_{a}, \tilde{b}_{a^{\prime}}\right)_{c}=0$ whenever $a \neq a^{\prime}$. Part $(a)$ guarantees that both $b_{0, a_{2}, \ldots, a_{\ell}}$ and $b_{0, a_{2}^{\prime}, \ldots, a_{\ell}^{\prime}}$ are in the kernel of $L$, so by Lemma 2.2 we obtain:

$$
\left(\tilde{b}_{a}, \tilde{b}_{a^{\prime}}\right)_{c} \in \delta_{a_{1}, a_{1}^{\prime}} \cdot\left(\tilde{b}_{\left(0, a_{2}, \ldots, a_{\ell}\right)}, b_{\left(0, a_{2}^{\prime}, \ldots, a_{\ell}^{\prime}\right)}\right)_{c} \cdot \mathbb{C}(c)=\delta_{a, a^{\prime}} \cdot \mathbb{C}(c)
$$

because the elements $b_{\left(0, a_{2}, \ldots, a_{\ell}\right)}$ and $b_{\left(0, a_{2}^{\prime}, \ldots, a_{\ell}^{\prime}\right)}$ of the canonical basis $\mathbf{B}$ are orthogonal unless $\left(a_{2}, \ldots, a_{\ell}\right)=\left(a_{2}^{\prime}, \ldots, a_{\ell}^{\prime}\right)$. This proves $(b)$.

Proposition 1.1 is proved. 
Proof of Theorem 1.3. Take a homogeneous set $u_{1}, \ldots, u_{\ell}$ of generators in $S(V)^{W}$, and denote, as usual, $u^{a} \stackrel{\text { def }}{=} u_{1}^{a_{1}} \cdots u_{\ell}^{a_{\ell}}$. By definition, $\left(u^{a}, e_{d_{k}}^{(c)}\right)_{c}=0$ for all $a \in \mathbb{Z}_{\geq 0}^{\ell}$ such that $a_{k}=\cdots=a_{\ell}=0$. For any such non-zero $a \in \mathbb{Z}_{\geq 0}^{\ell}$ let $s \leq k-1$ be the largest index such that $a_{s} \neq 0$ and let $a^{\prime} \stackrel{\text { def }}{=} a-\delta_{s} \in \mathbb{Z}_{\geq 0}^{\ell}$ (we denote $\delta_{s}=(0, \ldots, 1, \ldots, 0)$, where 1 is in the $s$-th position). Then $\left(u^{a^{\prime}}, \nabla_{u_{s}}\left(e_{d_{k}}^{(c)}\right)\right)_{c}=0$. In particular, the element $\nabla_{u_{s}}\left(e_{d_{k}}^{(c)}\right)$ is $(\cdot, \cdot)_{c}$-orthogonal to all of the monomials $u^{a^{\prime}}$ such that $\operatorname{deg} u^{a^{\prime}}=d_{k}-d_{s}$. Since the form $(\cdot, \cdot)_{c}$ is nondegenerate for generic $c$, this implies $\nabla_{u_{s}}\left(e_{d_{k}}^{(c)}\right)=0$.

By definition $e_{d_{k}}^{(c)} \equiv u_{k} \bmod S_{c}(V)_{\prec \delta_{k}}$ (see (3.1) and the property 1 of the canonical basis $b_{a}$ ), which implies that $\left(e_{d_{1}}^{(c)}\right)^{a_{1}} \ldots\left(e_{d_{\ell}}^{(c)}\right)^{a_{\ell}} \equiv u^{a} \bmod S_{c}(V)_{\prec a}$. Indeed, if for every $k=1, \ldots, h$

$$
e_{d_{k}}^{(c)}=u_{k}+\sum \beta_{p_{1}, \ldots, p_{k-1}} u_{1}^{p_{1}} \cdots u_{k-1}^{p_{k-1}}
$$

for some $\beta_{p_{1}, \ldots, p_{k-1}} \in \mathbb{C}(c)$ then

$$
\left(e_{d_{1}}^{(c)}\right)^{a_{1}} \ldots\left(e_{d_{\ell}}^{(c)}\right)^{a_{\ell}}=u_{1}^{a_{1}} \cdots u_{\ell}^{a_{\ell}}+\sum_{p_{1}, \ldots, p_{\ell-1}} \sum_{p_{\ell}=1}^{a_{\ell}-1} \gamma_{p_{1}, \ldots, p_{\ell}} u_{1}^{p_{1}} \cdots u_{\ell}^{p_{\ell}}
$$

for some $\gamma_{p_{1}, \ldots, p_{\ell}} \in \mathbb{C}(c)$. Here $\left(p_{1}, \ldots, p_{\ell}\right) \prec\left(a_{1}, \ldots, a_{\ell}\right)$, because the last non-zero coordinate of $\left(a_{1}-p_{1}, \ldots, a_{\ell}-p_{\ell}\right)$ is positive. Thus, the monomials $\left(e_{d_{1}}^{(c)}\right)^{a_{1}} \cdots\left(e_{d_{\ell}}^{(c)}\right)^{a_{\ell}}$ for all $a_{1}, \ldots, a_{\ell} \in \mathbb{Z}_{\geq 0}$ form a basis of $S_{c}(V)^{W}$, and part (a) of Theorem 1.3 is proved.

To prove part $(b)$ let $\mu_{k}^{(c)} \in S_{c}(V)^{W}$ be any element satisfying its conditions. Then for any $a \prec \delta_{k}$ one has $\left(u^{a}, \mu_{k}^{(c)}\right)_{c}=\nabla_{u_{1}}^{a_{1}} \cdots \nabla_{u_{k-1}}^{a_{k-1}}\left(\mu_{k}^{(c)}\right)=0$, hence $\mu_{k}^{(c)}=$ const $\cdot e_{d_{k}}^{(c)}+\sum_{\delta_{k} \prec q} \beta_{q} b_{q}$ for some $\beta_{q} \in \mathbb{C}(c)$. The element $b_{q}$ is homogeneous with $\operatorname{deg} b_{q}=\sum_{i=1}^{\ell} d_{i} q_{i}$. Since $d_{1}<\cdots<d_{k}<$ $\cdots<d_{\ell}$, one has $\operatorname{deg} b_{q}>d_{k}$ for every $q$. Therefore, the homogeneity condition implies that $\mu_{k}^{(c)}=$ const $\cdot e_{d_{k}}^{(c)}$. Part $(b)$ is proved.

Part $(c)$, given here for completeness, is proved in our previous paper [2].

Theorem 1.3 is proved.

\subsection{Proof of Theorem 1.4}

The argument follows almost literally the original proof for $c=0$ given in [15]; we put it here mostly for reader's convenience.

Abbreviate $\nabla_{x_{i}}$ as $\nabla_{i}$; also take $x \stackrel{\text { def }}{=}\left(x_{1}, \ldots, x_{n}\right)$ and $\nabla \stackrel{\text { def }}{=}\left(\nabla_{1}, \ldots, \nabla_{n}\right)$.

Let $c$ be generic.

Lemma 3.2. A function $f$ can be represented as $(P(\nabla))(\Delta(x))$ for some polynomial $P$ if and only if $\left(e_{k}(\nabla)\right)(f(x))=0$ for all $k$.

Proof. For $c=0$ (when $\nabla_{i}=\partial_{x_{i}}$ ) it is a theorem due to Steinberg [20]. For $c$ generic the Dunkl operators are conjugate to differentiations by means of some intertwining operator $B$ (see [11]): $\nabla_{i}=B^{-1} \partial_{x_{i}} B$, and therefore $e_{k}(\nabla)(f(x))=0$ is equivalent to $e_{k}(\partial) B(f(x))=0$. By the Steinberg's theorem it means that $f=B^{-1} P(\partial) \Delta=P(\nabla) B^{-1} \Delta$. But since $\Delta$ is skewsymmetric, $\nabla_{i} \Delta$ is proportional to $\partial_{x_{i}} \Delta$, and therefore $B^{-1} \Delta=\lambda \Delta$ for some constant $\lambda$. The lemma is proved.

Consider now the polynomials $f_{i}=\nabla_{i}\left(e_{n}^{(c)}\right)$. Obviously, $\left(e_{k}(\nabla)\right)\left(f_{i}\right)=0$ for all $k$, and therefore $f_{i}=\left(g_{i}(\nabla)\right)(\Delta(x))$. Without loss of generality, $g_{i}$ can be taken skew-invariant with respect to the subgroup $G_{i} \subset S_{n}$ of permutations leaving $i$ fixed; from degree considerations 
one obtains $g_{i}(x)=$ const $\cdot \Delta\left(x_{1}, \ldots, \widehat{x_{i}}, \ldots, x_{n}\right)$. Euler's formula $e_{n}^{(c)}=n \cdot \sum_{i=1}^{n} x_{i} f_{i}$ implies now (1.3) for $k=n$.

Let $p_{m}\left(y_{1}, \ldots, y_{s}\right) \stackrel{\text { def }}{=} y_{1}^{m}+\cdots+y_{s}^{m}$. Since (1.3) for $k=n$ is proved, one has

$$
\left(p_{i}\left(\nabla_{j_{1}}, \ldots, \nabla_{j_{k}}\right)\right)\left(f_{j_{1}, \ldots, j_{k}}(x)\right)=\text { const } \cdot \delta_{i k},
$$

where $1 \leq j_{1}<\cdots<j_{k} \leq n$ and $f_{j_{1}, \ldots, j_{k}} \stackrel{\text { def }}{=} e_{k}^{(c)}\left(x_{j_{1}}, \ldots, x_{j_{k}}\right)$. Thus, if $F_{k}$ is the right-hand side of (1.3), one has

$$
\begin{aligned}
p_{i}(\nabla) F_{k} & =\text { const } \cdot \sum_{1 \leq j_{1}<\cdots<j_{k} \leq n}\left(p_{i}(\nabla)\right)\left(f_{j_{1}, \ldots, j_{k}}(x)\right) \\
& =\text { const } \cdot \sum_{1 \leq j_{1}<\cdots<j_{k} \leq n}\left(p_{i}\left(\nabla_{j_{1}}, \ldots, \nabla_{j_{k}}\right)\right)\left(f_{j_{1}, \ldots, j_{k}}(x)\right)=\text { const } \cdot \delta_{i k},
\end{aligned}
$$

Hence, $F_{k}=e_{k}^{(c)}$.

Theorem 1.4 is proved.

\subsection{Proof of Theorem 1.5}

We need the following result.

Proposition 3.2. The canonical basis $\mathbf{B}$ is well-defined at $h_{c}=1($ e.g., at $c=1 / h)$ and $\mathbf{B} \backslash\{1\}$ is orthogonal with respect to the form $\bar{\Phi}_{c}$ (see Theorem 2.1).

Proof. Indeed, in the definition of $b_{a}$ in Theorem 1.1 we can take $u_{1}, \ldots, u_{\ell}$ to be a homogeneous generating set of $S\left(V_{\mathbb{R}}\right)_{+}^{W}$. Let us prove that for each $a \in \mathbb{Z}_{\geq 0}^{\ell} \backslash\{0\}$ the coefficients $c_{a, a^{\prime}}$ of the expansion $b_{a}=\sum_{a^{\prime} \preceq a} c_{a, a^{\prime}} u^{a}$ have no poles at $h_{c}=1$. Suppose the contrary. Then there exists an exponent $\ell>0$ such that $\tilde{b}_{a}=\left(1-h_{c}\right)^{\ell} b_{a}$ is regular at $h_{c}=1$ and $\pi\left(\tilde{b}_{a}\right) \neq 0$, where $\pi: \mathcal{A} \rightarrow \mathcal{A} /\left(1-h_{c}\right)$ is the canonical homomorphism ( $\mathcal{A}$ is as in Proposition 2.3). Since $\ell>0$, we see that $\pi\left(\tilde{b}_{a}\right) \in U \stackrel{\text { def }}{=} \sum_{a^{\prime} \prec a, a^{\prime} \neq 0} \mathbb{k} \cdot u^{a^{\prime}}$, where $\mathbb{k}=\mathcal{A} /\left(1-h_{c}\right)$. But since $\Phi_{c}\left(b_{a}, u^{a^{\prime}}\right)=0$ for all $a^{\prime} \prec a$, we see that $\bar{\Phi}_{c}\left(\pi\left(\tilde{b}_{a}\right), U\right)=0$ which contradicts Theorem 2.1(c). The contradiction obtained proves that $\ell=0$, i.e., $\pi\left(b_{a}\right)$ is well-defined. The orthogonality of these elements is obvious.

Let $W=S_{n}, V=\mathbb{C}^{n}$. Here $d_{k}=k, k=1, \ldots, n$. Recall that $e_{k}=e_{k}\left(x_{1}, \ldots, x_{n}\right) \in$ $\mathbb{C}\left[x_{1}, \ldots, x_{n}\right]$ is the $k$-th elementary symmetric polynomial and $\bar{e}_{k}=e_{k}\left(x_{1}-\frac{e_{1}(x)}{n}, \ldots, x_{n}-\frac{e_{1}(x)}{n}\right)$. The elements $\bar{e}_{k}, k=2, \ldots, n$ generate a subalgebra of $S(V)^{S_{n}}$ isomorphic to $S\left(V^{\prime}\right)^{S_{n}}$, where $V^{\prime}=\left\{x \in V \mid \sum_{i} x_{i}=0\right\}$.

Lemma 3.3. Using the notation $p_{r} \stackrel{\text { def }}{=} y_{1}^{r}+\cdots+y_{n}^{r}$, we obtain

$$
\nabla_{p_{r}}\left(\bar{e}_{k}\right)=(-1)^{r}(n-k+1) \cdots(n-k+r) \frac{(1-n c)^{r}-(1-n c)}{n^{r} c} \bar{e}_{k-r} .
$$

Proof. Denote

$$
Q_{k, i}(x) \stackrel{\text { def }}{=} e_{k}\left(x_{1}-\frac{e_{1}(x)}{n}, \ldots, x_{i}-\widehat{\frac{e_{1}}{(x}} \frac{1}{n}, \ldots, x_{n}-\frac{e_{1}(x)}{n}\right)
$$

(the $i$-th argument omitted). Easy calculations show that

$$
\sum_{i=1}^{n} Q_{k, i}=(n-k) \bar{e}_{k},
$$




$$
\begin{aligned}
& \frac{\partial Q_{k, i}}{\partial x_{i}}=-\frac{n-k}{n} Q_{k-1, i}, \\
& \frac{\partial \bar{e}_{k}}{\partial x_{i}}=\nabla_{y_{i}}\left(\bar{e}_{k}\right)=Q_{k-1, i}-\frac{n-k+1}{n} \bar{e}_{k-1} .
\end{aligned}
$$

Clearly,

$$
\sum_{j \neq i} \frac{(i j) Q_{k, i}-Q_{k, i}}{x_{i}-x_{j}}=(n-k) Q_{k-1, i},
$$

and therefore the Dunkl operator

$$
\nabla_{y_{i}}\left(Q_{k, i}\right)=(n-k)\left(c-\frac{1}{n}\right) Q_{k-1, i}
$$

An induction on $r$ (where (3.4) is the base) gives then

$$
\begin{aligned}
\nabla_{y_{i}}^{r}\left(\bar{e}_{k}\right)= & (n-k+1) \cdots(n-k+r-1) \frac{(n c-1)^{r}-(-1)^{r}}{n^{r} c} Q_{k-r, i} \\
& +(-1)^{r} \frac{(n-k+1) \cdots(n-k+r)}{n^{r}} \bar{e}_{k-r} .
\end{aligned}
$$

Summation over $i$ using (3.3) finishes the proof.

Lemma 3.3 implies that $\nabla_{p_{r}}^{(c)}\left(\bar{e}_{k}\right)=0$ for $c=1 / n$ and all $r<k$. Since $p_{1}, \ldots, p_{n}$ generate $\mathbb{C}\left[x_{1}, \ldots, x_{n}\right]^{S_{n}}$, we obtain

$$
\nabla_{P}^{(c)}\left(\bar{e}_{k}\right)=0
$$

for $c=1 / n$ and any homogeneous symmetric polynomial $P$ of degree $\operatorname{deg} P<k$.

On the other hand, by Theorem 1.3(b) one has $\nabla_{P}\left(e_{k}^{(c)}\right)=0$ for any homogeneous symmetric polynomial $P$ of degree $\operatorname{deg} P<k$. Therefore,

$$
\bar{\Phi}_{1 / n}\left(\bar{e}^{a^{\prime}}, \bar{e}_{k}\right)=0=\bar{\Phi}_{1 / n}\left(\bar{e}^{a^{\prime}}, e_{k}^{(1 / n)}\right)
$$

for any $a^{\prime} \prec \delta_{k}$. Here we abbreviated $\bar{\Phi}_{1 / n} \stackrel{\text { def }}{=} \bar{\Phi}_{c}$ with $c=1 / n$ in the notation of Theorem 2.1 and $e_{k}^{(1 / n)} \stackrel{\text { def }}{=} \lim _{c \rightarrow 1 / h} e_{k}^{(c)}$, which is well-defined by Proposition 3.2. Consequently,

$$
\bar{\Phi}_{1 / n}\left(U, e_{k}^{(1 / n)}-\alpha \bar{e}_{k}\right)=0
$$

for all $a^{\prime} \prec \delta_{k}, \alpha \in \mathbb{C}^{\times}$, where $U=\sum_{a^{\prime} \prec \delta_{k}} \mathbb{C} \cdot \bar{e}^{a^{\prime}}$. Therefore, $e_{k}^{(1 / n)}=\alpha \bar{e}_{k}$ because, on the one hand, $e_{k}^{(1 / n)}-\alpha \bar{e}_{k} \in U$ for some $\alpha \neq 0$, and on the other hand, the restriction of $\bar{\Phi}_{1 / n}$ to $U$ is non-degenerate by Theorem 2.1(c).

Theorem 1.5 is proved.

\subsection{Canonical invariants of dihedral groups and proof of Theorem 1.2}

Throughout the section we deal with the dihedral group

$$
W=I_{2}(m)=\left\langle s_{0}^{2}=s_{1}^{2}=\left(s_{0} s_{1}\right)^{m}=1\right\rangle
$$

of order $2 m$. 
We denote by $\{z, \bar{z}\}$ the basis of $V$ such that

$$
s_{j}(z)=-\zeta^{j} \bar{z}, \quad s_{j}(\bar{z})=-\zeta^{-j} z .
$$

for $j=0,1$, where $\zeta=e^{2 \pi i / m}$ is an $m$-th primitive root of unity.

We also denote by $e_{2}, e_{m}$ the generators of $S(V)^{W}$ given by

$$
e_{2}=z \bar{z}, \quad e_{m}=z^{m}+\bar{z}^{m} \text {. }
$$

Lemma 3.4. The restriction of the Dunkl Laplacian $L$ to $S(V)^{W}=\mathbb{C}\left[e_{2}, e_{m}\right]$ for $W=I_{2}(m)$ equals:

$$
L=e_{2} \partial_{e_{2}}^{2}+m e_{m} \partial_{e_{2}} \partial_{e_{m}}+m^{2} e_{2}^{m-1} \partial_{e_{m}}^{2}+\left(1-\frac{m}{2} C\right) \partial_{e_{2}}+\frac{m^{2}}{2} \delta e_{2}^{m / 2-1} \partial_{e_{m}}
$$

where $C \stackrel{\text { def }}{=} c\left(s_{1}\right)+c\left(s_{2}\right)$ and $\delta \stackrel{\text { def }}{=} c\left(s_{2}\right)-c\left(s_{1}\right)$ (so that $\delta=0$ when $m$ is odd); $\partial_{e_{2}}$ and $\partial_{e_{m}}$ mean here differentiation with respect to $e_{2}$ and $e_{m}$, respectively, in the ring $\mathbb{C}\left[e_{2}, e_{m}\right]$.

Proof. Clearly, $s \circ \partial_{y}=\partial_{s^{*}(y)} \circ s$ for any linear automorphism $s$ of $V$ and any $y \in V^{*}$, where $\partial_{y}: S(V) \rightarrow S(V)$ is the directional derivative. So, $L$ can be rewritten in the form $L=\sum_{i} D_{i} s_{i}$ where $D_{i}$ are differential operators (of order at most 2) and $s_{i}$ are reflections. Thus, on the space of invariant functions $L$ is a second order differential operator. To determine its coefficients it suffices to compute $L$ on monomials of degree 1 and 2 in $e_{2}, e_{m}$. This is done in [10]; see also [2].

Corollary 3.1. In the notation of Lemma 3.4, we have

$$
\frac{4}{m^{2}} e_{2} L=\mathcal{E}^{2}-C \mathcal{E}+\left(4 \frac{e_{2}^{m}}{e_{m}^{2}}-1\right)\left(\mathcal{D}^{2}-\mathcal{D}\right)+\left(C-1+2 \delta \frac{e_{2}^{m / 2}}{e_{m}}\right) \mathcal{D}
$$

where $\mathcal{E} \stackrel{\text { def }}{=} \frac{2}{m} e_{2} \partial_{e_{2}}+e_{m} \partial_{e_{m}}$ is a multiple of the Euler derivation, and $\mathcal{D} \stackrel{\text { def }}{=} e_{m} \partial_{e_{m}}$.

Proof. Since $\partial_{e_{2}} e_{2}=e_{2} \partial_{e_{2}}+1$, one obtains

$$
4 e_{2} L=\left(2 e_{2} \partial_{e_{2}}\right)^{2}+4 m\left(e_{2} \partial_{e_{2}}\right)\left(e_{m} \partial_{e_{m}}\right)+4 m^{2} e_{2}^{m} \partial_{e_{m}}^{2}-2 m C e_{2} \partial_{e_{2}}+2 m^{2} \delta e_{2}^{m / 2} \partial_{e_{m}} .
$$

Equalities $\left(2 e_{2} \partial_{e_{2}}\right)^{2}+4 m\left(e_{2} \partial_{e_{2}}\right)\left(e_{m} \partial_{e_{m}}\right)=m^{2}\left(\mathcal{E}^{2}-\mathcal{D}^{2}\right)$ and $\partial_{e_{m}}^{2}=\frac{1}{e_{m}^{2}}\left(\mathcal{D}^{2}-\mathcal{D}\right)$ imply now that

$$
\frac{4}{m^{2}} e_{2} L=\mathcal{E}^{2}-\mathcal{D}^{2}+4 \frac{e_{2}^{m}}{e_{m}^{2}}\left(\mathcal{D}^{2}-\mathcal{D}\right)-C(\mathcal{E}-\mathcal{D})+2 \delta \frac{e_{2}^{m / 2}}{e_{m}} \mathcal{D},
$$

and the corollary follows.

Corollary 3.2 (of Corollary 3.1). Let $f=f(x, u) \in \mathbb{C}[[x, u]]$. Then

$$
\frac{4}{m^{2}} e_{2} L\left(f\left(\frac{e_{m}}{e_{2}^{m / 2}}, e_{2}^{m / 2} t\right)\right)=\left.L_{x, u}(f(x, u))\right|_{x=\frac{e_{m}}{e_{2}^{m / 2}}, u=e_{2}^{m / 2} t}
$$

where

$$
L_{x, u}=u^{2} \partial_{u}^{2}-(C-1) u \partial_{u}+\left(4-x^{2}\right) \partial_{x}^{2}+((C-1) x+2 \delta) \partial_{x} .
$$


Proof. Follows from

$$
\mathcal{D}\left(f\left(\frac{e_{m}}{e_{2}^{m / 2}}, e_{2}^{m / 2} t\right)\right)=\frac{e_{m}}{e_{2}^{m / 2}} f_{x}\left(e_{m} / e_{2}^{m / 2}, e_{2}^{m / 2} t\right)=\left.\left(x \partial_{x}\right) f(x, u)\right|_{x=\frac{e_{m}}{e_{2}^{m / 2}}, u=e_{2}^{m / 2} t}
$$

and

$$
\begin{aligned}
\mathcal{E}\left(\left(\frac{e_{m}}{e_{2}^{m / 2}}\right)^{p}\left(e_{2}^{m / 2} t\right)^{q}\right) & =\left(\frac{2}{m} e_{2} \partial_{e_{2}}+e_{m} \partial_{e_{m}}\right)\left(e_{m}^{p} e_{2}^{(q-p) m / 2} t^{q}\right) \\
& =q\left(e_{m}^{p} e_{2}^{(q-p) m / 2} t^{q}\right)=\left.\left(u \partial_{u}\right)\left(x^{p} u^{q}\right)\right|_{x=\frac{e_{m}}{e_{2}^{m / 2}}, u=e_{2}^{m / 2} t}
\end{aligned}
$$

Proof of Theorem 1.2. In view of Corollary 3.2, for $c=$ const it suffices to prove that $L_{x, u}\left(p^{c}\right)=0$, where $p=1+x u+u^{2}$. Indeed,

$$
\begin{aligned}
& u \partial_{u}\left(p^{c}\right)=c u(x+2 u) p^{c-1}=2 c p^{c}-c(2+u x) p^{c-1}, \\
& \left(\left(u \partial_{u}\right)^{2}-2 c u \partial_{u}\right)\left(p^{c}\right)=u \partial_{u}\left(u \partial_{u}-2 c\right)\left(p^{c}\right)=-c u \partial_{u}\left((2+u x) p^{c-1}\right) \\
& =-\operatorname{cuxp}^{c-1}-c(c-1) u(2+u x)(x+2 u) p^{c-2}, \\
& \partial_{x}\left(p^{c}\right)=c u p^{c-1}, \\
& \partial_{x}^{2}\left(p^{c}\right)=c(c-1) u^{2} p^{c-2} .
\end{aligned}
$$

For $c=$ const one has $C=2 c$ and $\delta=0$, so that

$$
\begin{aligned}
c^{-1} p^{2-c} L_{x, u}\left(p^{c}\right) & =-u x p-(c-1) u(2+u x)(x+2 u)+\left(4-x^{2}\right)(c-1) u^{2}+(2 c-1) x u p \\
& =(c-1) u\left(2 x p-(2+u x)(x+2 u)+\left(4-x^{2}\right) u\right) \\
& =0 .
\end{aligned}
$$

This proves part $(a)$ of Theorem 1.2.

To prove part $(b)$ define $I_{r}^{(a, b)}(y, u) \in \mathbb{C}[[y, u]]$ for each $r, a, b$ by

$$
I_{r}^{(a, b)}=\int_{0}^{1} s^{a+b}(1-s)^{-b-1-r}\left(1-s+u s\left(1-\frac{s}{2}(1-y)\right)\right)^{r} d s .
$$

Clearly, the right-hand side of (1.2) equals $I_{r}^{(a, b)}(y, u)$ with $a=-(C+\delta+1) / 2, b=-(C-\delta+1) / 2$, $r=-b-1, u=e_{2}^{m / 2} t$, and $y=\frac{e_{m}}{2 e_{2}^{m / 2}}$ (so that $\left.\frac{1-y}{2}=-\frac{e_{m}^{\prime}}{e_{2}^{m / 2}}\right)$.

Therefore, in view of Corollary 3.2, it suffices to prove that

$$
L_{x, u}\left(I_{r}^{(a, b)}(x / 2, u)\right)=0
$$

for all $r$, where $a, b$ are as above, and to determine the normalizing coefficients $n_{k}(c)$.

Recall that the $n$-th Jacobi polynomial $P_{n}^{(a, b)}(y)$ is given by

$$
P_{n}^{(a, b)}(y)=\frac{\Gamma(a+n+1)}{n ! \Gamma(n+a+b+1) \Gamma(-n-b)} \int_{0}^{1} s^{n+a+b}(1-s)^{-n-b-1}\left(1-\frac{s}{2}(1-y)\right)^{n} d s
$$

(with the analytic continuation to all $a, b \in \mathbb{C}$ ). Thus one has

$$
\begin{aligned}
I_{r}^{(a, b)}(y, u) & =\int_{0}^{1} s^{a+b}(1-s)^{-b-1} \sum_{n=0}^{\infty} \frac{\Gamma(r+1)}{n ! \Gamma(r-n+1)} u^{n} s^{n}(1-s)^{-n}\left(1-\frac{s}{2}(1-y)\right)^{n} d s \\
& =\sum_{n=0}^{\infty} \frac{\Gamma(r+1)}{n ! \Gamma(r-n+1)} u^{n} \int_{0}^{1} s^{a+b+n}(1-s)^{-b-1-n}\left(1-\frac{s}{2}(1-y)\right)^{n} d s
\end{aligned}
$$




$$
\begin{aligned}
& =\sum_{n=0}^{\infty} \frac{\Gamma(r+1) \Gamma(n+a+b+1) \Gamma(-n-b)}{\Gamma(r-n+1) \Gamma(a+n+1)} P_{n}^{(a, b)}(y) u^{n} \\
& \stackrel{\text { def }}{=} \sum_{n=0}^{\infty} q_{n}(r, a, b) P_{n}^{(a, b)}(y) u^{n} .
\end{aligned}
$$

The Jacobi polynomial $P_{n}^{(a, b)}(y)$ belongs to the kernel of the differential operator

$$
J^{(a, b)} \stackrel{\text { def }}{=}\left(1-y^{2}\right) \partial_{y}^{2}+(b-a-(a+b+2) y) \partial_{y}+n(n+a+b+1)
$$

(see e.g. [1] for proof). Therefore, $I^{(a, b)}(y, u)=\sum_{n=0}^{\infty} q_{n}(r, a, b) P_{n}^{(a, b)}(y) u^{n}$ satisfies

$$
\tilde{L}_{y, u}^{(a, b)}\left(I_{r}^{(a, b)}(y, u)\right)=0
$$

where $\tilde{L}_{y, u}^{(a, b)}=\left(1-y^{2}\right) \partial_{y}^{2}+(b-a-(a+b+2) y) \partial_{y}+u^{2} \partial_{u}^{2}+(a+b+2) u \partial_{u}$.

Take now $a=-(C+\delta+1) / 2, b=-(C-\delta+1) / 2$, so that $a+b+2=-(C-1), b-a=\delta$. One has then

$$
\begin{aligned}
\tilde{L}_{y, u}^{(a, b)} & =\left(1-y^{2}\right) \partial_{y}^{2}+(\delta+(C-1) y) \partial_{y}+u^{2} \partial_{u}^{2}-(C-1) u \partial_{u} \\
& =\left(4-(2 y)^{2}\right) \partial_{2 y}^{2}+(2 \delta+(C-1)(2 y)) \partial_{2 y}+u^{2} \partial_{u}^{2}-(C-1) u \partial_{u} \\
& =L_{2 y, u} .
\end{aligned}
$$

Therefore, (3.8) implies (3.6).

To finish the proof it remains to find the value of the normalization coefficients $n_{k}=n_{k}(c)$ in (1.2). To do this, substitute $e_{2}=0$ into (1.2), so that $e_{m}^{\prime}=e_{m} / 4$. Under this specialization, $b_{(0, k)}$ becomes a (complex) multiple of $e_{m}^{k}$ and the right-hand side of (1.2) becomes (with the abbreviation $\left.\alpha=\frac{C-\delta-1}{2}\right)$ :

$$
\begin{aligned}
\int_{0}^{1}(1 & -s)^{\alpha} s^{-C-1}\left(1+\frac{t s^{2}}{4(1-s)} e_{m}\right)^{\alpha} d s \\
& =\sum_{k=0}^{\infty}\left(\begin{array}{l}
\alpha \\
k
\end{array}\right) t^{k}\left(\frac{e_{m}}{4}\right)^{k} \int_{0}^{1}(1-s)^{\alpha-k} s^{-C+2 k-1} d s \\
& =\sum_{k=0}^{\infty}\left(\begin{array}{l}
\alpha \\
k
\end{array}\right) \frac{\Gamma(\alpha-k+1) \Gamma(-C+2 k)}{\Gamma(\alpha+k-C+1)} t^{k}\left(\frac{e_{m}}{4}\right)^{k} \\
& =\sum_{k=0}^{\infty} \frac{\Gamma(\alpha+1) \Gamma(-C+2 k)}{k ! \cdot \Gamma(\alpha+k-C+1)} t^{k}\left(\frac{e_{m}}{4}\right)^{k} \\
& =\sum_{k=0}^{\infty} \frac{\Gamma\left(\frac{C-\delta+1}{2}\right) \Gamma(2 k-C)}{\Gamma\left(k-\frac{C+\delta-1}{2}\right)} t^{k} \frac{e_{m}^{k}}{4^{k} k !} .
\end{aligned}
$$

This proves (1.2) and finishes the proof of part (b) of Theorem 1.2.

\section{Acknowledgments}

The work was partially supported by the CRDF grant RUM1-2895-MO-07. The second author was also supported by the INTAS grant 05-7805, RFBR grants 08-01-00110-a and NSh709.2008.1, and the HSE Scientific Foundation grant 08-01-0019.

The authors are grateful to P. Etingof, M. Feigin, A. Samokhin, and Y. Xu for valuable discussions. The second author wishes to thank the University of Oregon, where most of this work was carried out, for its warm hospitality. 


\section{References}

[1] Andrews G.E., Askey R., Roy R., Special functions, Encyclopedia of Mathematics and Its Applications, Vol. 71, Cambridge University Press, Cambridge, 1999.

[2] Berenstein A., Burman Yu., Quasiharmonic polynomials for Coxeter groups and representations of Cherednik algebras, Trans. Amer. Math. Soc., to appear, math.RT/0505173.

[3] Berest Yu., The problem of lacunas and analysis on root systems, Trans. Amer. Math. Soc. 352 (2000), 3743-3776.

[4] Berest Y., Etingof P., Ginzburg V., Finite-dimensional representations of rational Cherednik algebras, Int. Math. Res. Not. 2003 (2003), no. 19, 1053-1088, math.RT/0208138.

[5] Broue M., Malle G., Rouquier R., Complex reflection groups, braid groups, Hecke algebras, J. Reine Angew. Math. 500 (1998), 127-190.

[6] Chevalley C., Invariants of finite groups generated by reflections, Amer. J. Math. 77 (1955), 778-782.

[7] Cohen A., Finite complex reflection groups, Ann. Sci. École Norm. Sup. (4) 9 (1976), 379-436.

[8] Dunkl C.F., Differential-difference operators associated to reflection groups, Trans. Amer. Math. Soc. 311 (1989), 167-183.

[9] Dunkl C.F., Intertwining operators and polynomials associated with symmetric group, Monatsh. Math. 126 (1998), 181-209.

[10] Dunkl C.F., Polynomials associated with dihedral groups, SIGMA 3 (2007), 052, 19 pages, math.CA/0702107.

[11] Dunkl C.F, de Jeu M.F.E., Opdam E.M., Singular polynomials for finite reflection groups, Trans. Amer. Math. Soc. 346 (1994), 237-256.

[12] Dunkl C.F., Xu Yu., Orthogonal polynomials of several variables, Cambridge University Press, Cambridge, 2001.

[13] Etingof P., Stoica E., Griffeth S., Unitary representations of rational Cherednik algebras, arXiv:0901.4595.

[14] Heckman G.J., A remark on the Dunkl differential-difference operators, in Harmonic analysis on reductive groups (Brunswick, ME, 1989), Progr. Math., Vol. 101, Birkhäuser Boston, Boston, MA, 1991, $181-191$.

[15] Iwasaki K., Basic invariants of finite reflection groups, J. Algebra 195 (1997), 538-547.

[16] Kostant B., Clifford algebra analogue of the Hopf-Koszul-Samelson theorem, the $\varrho$-decomposition $\mathcal{C}(\mathfrak{g})=$ End $V_{\varrho} \otimes \mathcal{C}(P)$, and the $\mathfrak{g}$-module structure of $\bigwedge \mathfrak{g}, A d v$. Math. 125 (1997), 275-350.

[17] Lapointe L., Lascoux A., Morse J., Determinantal expression and recursion for Jack polynomials, Electron. J. Combin. 7 (2000), Note 1, 7 pages.

[18] Macdonald I., Affine Hecke algebras and orthogonal polynomials, Cambridge Tracts in Mathematics, Vol. 157, Cambridge University Press, Cambridge, 2003.

[19] Opdam E., Harmonic analysis for certain representations of graded Hecke algebras, Acta Math. 175 (1995), $75-121$.

[20] Steinberg R., Differential equations invariant under finite reflection groups, Trans. Amer. Math. Soc. 112 (1964), 392-400. 